\title{
Tumor Odontogénico Epitelial Calcificante maxilar superior: reporte de un caso.
}

\section{Calcifying Epithelial Odontogenic Tumor in upper maxillary: a case report.}

\begin{abstract}
Carolina Ortega $^{1^{\star}}$, Cristián Núñez ${ }^{1,2}$, Guillermo Quezada ${ }^{1,2}$, Iris Espinoza ${ }^{3}$, Tomás Donoso ${ }^{4}$
1. Académico (a), Departamento de Cirugía y Traumatología Máxilofacial, Facultad de Odontología, Universidad de Chile.Chile

2. Cirujano-máxilofacial, Servicio de Cirugía de Cabeza y Cuello, Hospital Barros Luco-Trudeau. Chile

3. Patóloga Buco máxilofacial, Departamento de Patología y Medicina Oral. Facultad de Odontología de la Universidad de Chile. Servicio de Anatomía Patológica, Hospital Barros Luco-Trudeau. Chile 4. Becado Cirugía Maxilofacial, Universidad de Chile. Chile

* Correspondencia autor. Dra. Carolina Ortega Sobarzo | Dirección: Av. José Miguel Carrera 3204,

\section{RESUMEN}

El Tumor Odontogénico Epitelial Calcificante (TOEC), también denominado Tumor de Pindborg, se define como una neoplasia benigna, caracterizada por la proliferación epitelial; presenta secreción de una proteína tipo amiloide con tendencia a la calcificación. Representa menos del $1 \%$ de los tumores odontogénicos. Reportamos un caso en paciente de 75 años con un TOEC en la región maxilar izquierda en relación a una pieza dentaria incluida. El caso no mostró sintomatología específica, sólo una expansión de corticales vestibular y palatina. El diagnóstico se confirmó histológicamente mediante biopsia incisional y extirpación quirúrgica de la lesión, mostrando una histomorfología clásica para este tipo de lesiones. Reportamos este caso para resaltar la edad de presentación inusual, especialmente su localización e ilustrar su abordaje terapéutico y seguimiento.
\end{abstract} San Miguel, Región Metropolitana, Chile | E-mail: carolina.ortega.sobarzo@gmail.com | Celular:+56 977765019

Trabajo recibido el 18/03/2018.

Aprobado para su publicación el 14/12/2018
PALABRAS CLAVE:

Tumores odontogénicos, Tumor de Pindborg, Tumor odontogénico epitelial calcificante, Neoplasia epitelial.

Rev. Clin. Periodoncia Implantol. Rehabil. Oral Vol. 12(2); 100-102, 2019.

\section{ABSTRACT}

The Calcifying Epithelial Odontogenic Tumor (CEOT), also called Pindborg Tumor, is defined as a benign neoplasm, characterized by epithelial proliferation; secretion of an amyloid-like protein is present with a tendency to calcification. It represents less than $1 \%$ of odontogenic tumors. We report a case of a 75 -year-old patient with a TOEC in the left maxillary region in relation to an included tooth. The case did not show specific symptomatology, only an expansion of vestibular and palatal corticals.

The diagnosis was confirmed histologically by incisional biopsy and surgical removal of the lesion, showing a classic histomorphology for this type of lesions. We report this case to highlight the unusual age of presentation, especially its location and to illustrate its therapeutic approach and follow-up.

KEY WORDS:

Odontogenic tumors, Pindborg tumor, Calcifying epithelial odontogenic tumor, Epithelial neoplasm.

Rev. Clin. Periodoncia Implantol. Rehabil. Oral Vol. 12(2); 100-102, 2019

\section{INTRODUCCIÓN}

El Tumor Odontogénico Epitelial Calcificante (TOEC), también denominado Tumor de Pindborg, se define como una neoplasia benigna, caracterizada por la proliferación epitelial; presentando secreción de una proteína tipo amiloide con tendencia a la calcificación. Es muy inusual, representando menos del $1 \%$ de los tumores odontogénicos. El promedio de edad en el momento del diagnóstico es de 40 años, siendo afectados ambos sexos por igual. La recurrencia reportada es cercana a un $15 \%(1,2)$.

Se han descrito dos variantes clínico-radiográficas, una intraósea, la más común, y otra periférica que representaría el $6 \%$ de los casos. A nivel intraóseo, la localización más común es zona de premolares y molares mandibulares, y en un $52 \%$ de los casos asociado a un molar incluido ${ }^{(3)}$.

En su histopatología esta neoplasia se caracteriza por la proliferación de células epiteliales poliédricas con pleomorfismo nuclear, y presencia de una secreción tipo amiloide y calcificaciones ${ }^{(3)}$. Variantes de TOEC no calcificantes ${ }^{(4)}$ y con células claras ${ }^{(5)}$ se asociarían con un comportamiento más agresivo ${ }^{(6,7)}$. La caracterización de la proteína amiloide secretada por las células neoplásicas, denominada en forma provisoria AODAM, es un avance reciente ${ }^{(8)}$.

Respecto a su histogénesis, el primer reporte de esta entidad patológica realizada por Pindborg ${ }^{(9)}$ en el año 1958 menciona que derivaría de los epitelios reducidos del órgano del esmalte; actualmente se atribuye que sería de los remanentes de la lámina dental(3).

Se presenta un caso de TOEC, discutiendo su diagnóstico clínicoradiográfico diferencial y abordaje terapéutico.

\section{INFORME DEL CASO}

Paciente género masculino, 75 años de edad, derivado desde un Centro de Atención Primaria de Salud consulta en Servicio de Cirugía Maxilofacial del Hospital Barros Luco-Trudeau (HBLT) por aumento de volumen hemimaxilar izquierdo. Refiere consultas previas para tratamiento protésico en maxilar superior. Entre sus antecedentes mórbidos destaca hipertensión en tratamiento con Enalapril y Ácido Acetil Salicílico. Al examen extraoral se observa asimetría facial con comisura labial izquierda caudal respecto 
a contralateral, sin aumento de volumen ni cambio de coloración en la piel, dinámica mandibular normal, sin adenopatías palpables. Al examen intraoral, desdentado parcial, aumento de volumen de consistencia dura localizado en la zona del reborde maxilar izquierdo con expansión de corticales vestibular y palatina. La mucosa oral de recubrimiento es normal (Figura 1). En la ortopantomografía se observa un área de forma redondeada con densidad mixta, bien delimitada, ubicada en zona reborde maxilar izquierdo. La pieza dentaria 2.8 se encuentra en mesioversión y se proyecta sobre el borde distal de la lesión (Figura 2).

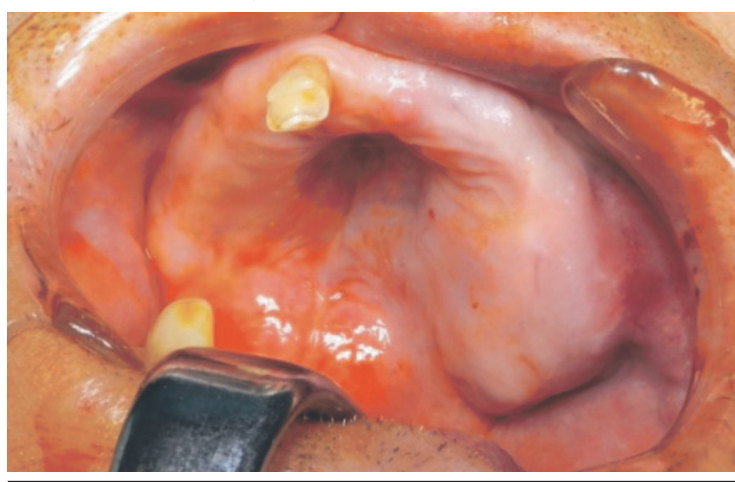

Figura 1. Imagen preoperatoria, se observa la expansión de corticales vestibular y palatina.

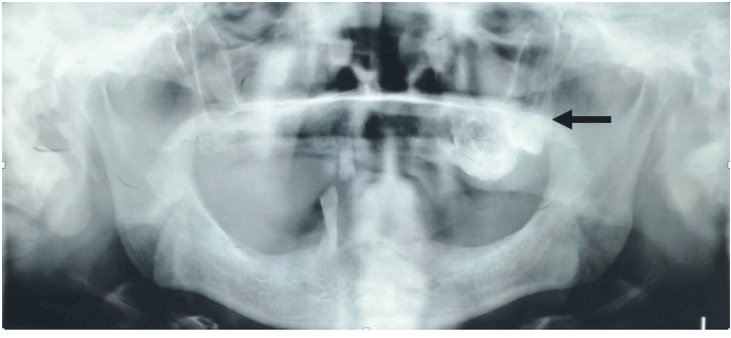

Figura 2. Ortopantomografía preoperatoria, se observa área de densidad mixta en relación a sector posterior de maxilar izquierdo, incluyendo pieza dentaria 2.8

A la tomografía computarizada se observa lesión redondeada, bien delimitada, con zonas hiperdensas e hipodensas irregulares en su interior. Al corte coronal se evidencia su proyección desde reborde maxilar izquierdo, corte axial muestra que expande corticales (Figura 3a y $3 \mathrm{~b}$ ). Con los hallazgos obtenidos se plantea como hipótesis diagnóstica fibroma osificante y osteoma.

Bajo anestesia local, se realiza biopsia incisional en surco vestibular a nivel de la zona del tercer molar, con muestra de ventana ósea, enviándose a estudio histopatológico, obteniéndose el diagnóstico de TOEC.

El paciente fue sometido en un segundo tiempo, bajo anestesia general, a una maxilectomía parcial y remodelación del lecho quirúrgico (Figura 4). La pieza quirúrgica fue enviada a estudio histopatológico, mostrando similares características a la biopsia incisional, presencia de islas de células poliédricas, de núcleos pleomórficos, con citoplasma eosinofílico y puentes intercelulares bien desarrollados. Presencia de estructuras basófilas mineralizadas de forma redondeada o que se fusionan, algunos como anillos concéntricos basófilos llamados anillos de Liesegang y zonas eosinófilas compatibles con material tipo amiloide (Figura 5). Al control post operatorio de 6 meses se aprecia evolución favorable mostrando reparación ósea y ausencia de recurrencia (Figura 6 y 7 ).

\section{DISCUSIÓN}

El presente caso, concuerda con las características clínicas descritas para esta neoplasia odontogénica, como son crecimiento lento, expansión de corticales y asociación con dientes incluidos, presente en aproximadamente el $50 \%$ de los $\operatorname{casos}^{(1,2,3)}$, sin embargo resulta interesante de reportar especialmente por su localización, debido a que la ubicación en maxilar es menos frecuente, presentándose principalmente a nivel intraóseo y predominantemente en zona mandibular, en una relación de 2:1 mandíbula: maxila ${ }^{(3)}$; y frecuentemente asociado a piezas dentarias incluidas u odontomas ${ }^{(2,3)}$. En aquellos casos que hay afectación del maxilar superior, los pacientes pueden manifestar sintomatología atribuida a la compresión mecánica de estructuras vecinas.

Cuando se afecta el seno maxilar, puede presentar congestión u obstrucción nasal, cefalea y epistaxis ${ }^{(10,11)}$. Al momento del diagnóstico solo

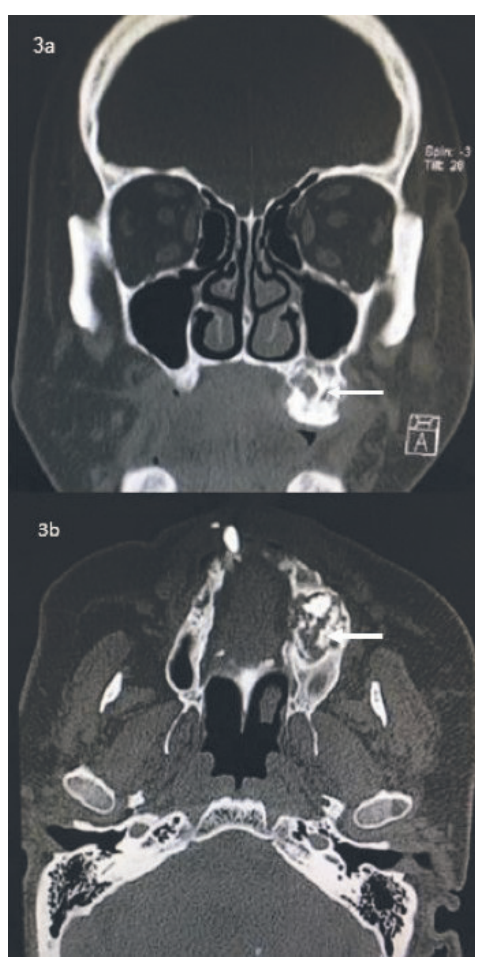

Figura 3. 3a: Corte coronal de tomografía computada, donde se observan zonas hiperdensas e hipodensas irregulares en su interior delimitada, en relación a tuberosidad izquierda. 3b: Corte axial de tomografía computada, donde se observa expansión de corticales vestibular y palatina.

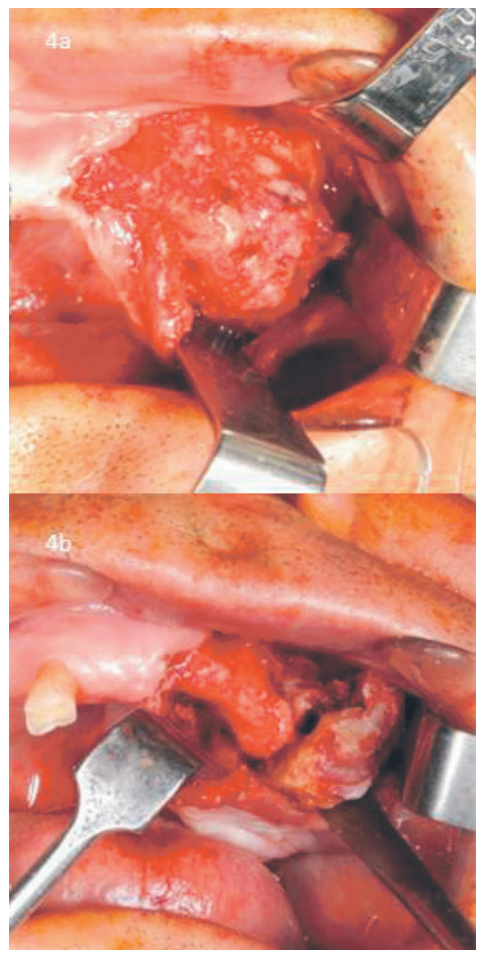

Figura 4. a y b: Imagen intraoperatoria, se observa exéresis de lesión con márgenes libres de lesión.

se presentaba compromiso del piso del seno maxilar, sin proyección hacia su interior. Respecto a la edad de presentación, el TOEC se diagnostica comúnmente en pacientes de mediana edad, entre tercera y sexta década, preferentemente a los 40 años, sin predilección por sexo(1). El caso reportado presenta una edad inusual de presentación, siendo mayor que lo reportado en la literatura, lo que podría explicarse por su crecimiento lento y asintomático. Radiográficamente el TOEC puede presentar una radiolucidez uni o multilocular, el caso reportado fue de densidad mixta, áreas radiolúcidas con calcificaciones en su interior, lo que es descrito en 


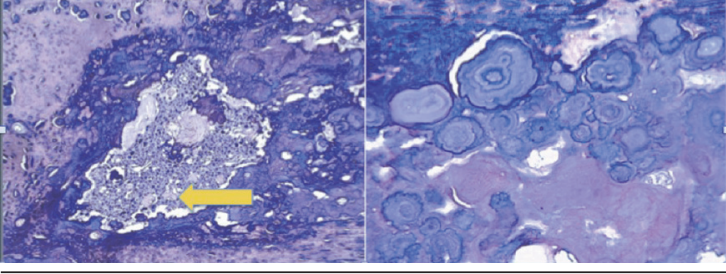

Figura 5. Ambas imágenes muestran Tinción de rojo Congo,(izquierda $x$ 40 y la derecha $x$ 100), de pieza quirúrgica extraída, donde se observa presencia de material amiloide.

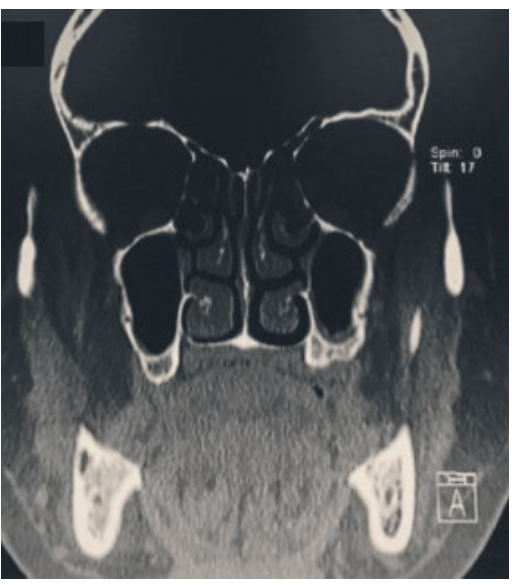

Figura 6. Corte coronal de tomografía computada, donde se observa reparación ósea en relación a zona intervenida.

la literatura como "driven snow"(12). Estos tumores pasarían por 3 etapas, en un comienzo es totalmente radiolúcido, luego aparecen algunas

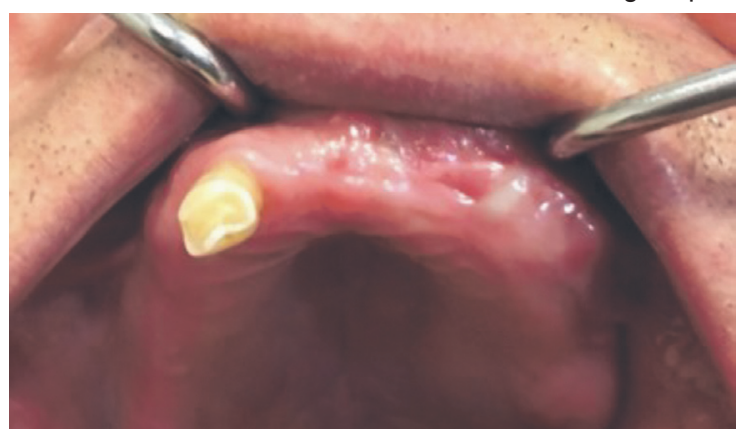

Figura 7. Imagen postoperatoria a 6 meses de realizada la cirugía de resección. Se observa reparación de los tejidos, sin recidivas actuales de la lesión. calcificaciones intratumorales, siendo la imagen más característica, aunque no patognomónica ${ }^{(13)}$. Existen otras lesiones de densidad mixta que fueron planteadas como diagnósticos diferenciales por sus características de área bien delimitada y de densidad mixta, lesión fibroósea del tipo fibroma osificante, neoplasia ósea benigna que también presenta bordes bien delimitados, pero que se produce principalmente en mujeres, tercera y cuarta década de vida y con mayor frecuencia en mandíbula. La posibilidad de un osteoma u otra neoplasia benigna fue sugerida, sin embargo, los osteomas son eminentemente radiopacos y se diagnostican de preferencia en hombres, en tamaños menores a 2 centímetros y zona mandibular. El osteoblastoma, otra neoplasia ósea benigna se caracteriza por presentar sintomatología dolorosa y áreas radiolúcidas y radiopacas pero sin un borde esclerótico ${ }^{(1)}$.

El tratamiento del TOEC depende del tamaño y localización anatómica. Aquellos tumores menores a 4 centímetros, que poseen bordes definidos localizados tanto en maxilar como en mandíbula, se tratan mediante enucleación o resección marginal con un margen de tejido óseo sano. En el caso de tumores de mayor tamaño la alternativa quirúrgica es la resección en bloque con una reconstrucción posterior. La base del tratamiento quirúrgico es obtener márgenes quirúrgicos sanos, tanto en tejido óseo como blando, requiriendo confirmación histopatológica posterior ${ }^{(14)}$. De acuerdo a las dimensiones del presente caso, se decidió tratar mediante maxilectomía parcial y posterior remodelación del lecho quirúrgico. En la tomografía computarizada postoperatoria a los 6 meses, se observa reparación ósea en el sitio quirúrgico mostrando una evolución favorable y ausencia de recidivas.

La histopatología del caso muestra islotes de células epiteliales poliédricas con pleomorfismo nuclear, nucléolos prominentes y puentes intercelulares. Es importante recordar que este pleomorfismo no se asocia con malignidad, existiendo la posibilidad que especialistas no familiarizados con esta entidad sospechen de una neoplasia maligna por error. Dentro de las características distintivas a nivel microscópico, se encuentra la presencia de áreas acelulares tipo amiloide dentro o adyacentes a islas epiteliales ${ }^{(3)}$; material que se demuestra mejor con tinción de rojo Congo(15). En la actualidad se ha identificado que este material posee una proteína única que tiene similitudes con las proteínas del esmalte ${ }^{(12,15)}$. Otra de las características histológicas distintivas de esta neoplasia, es la presencia de los anillos de Leisegang, los cuáles son anillos concéntricos de calcificaciones basófilas, dentro de esta masa de material amiloide ${ }^{(15)}$, que explica las características imagenológicas de esta neoplasia.

El seguimiento a los 6 meses muestra una evolución favorable, con favorable reparación ósea del sitio intervenido.

\section{CONCLUSIONES}

El caso reportado corresponde a un Tumor Odontogénico Epitelial Calcificante, el cuál fue diagnosticado mediante biopsia incisional y confirmado posteriormente con el análisis de la muestra de la excisión quirúrgica completa. Esta entidad corresponde a una neoplasia odontogénica sumamente infrecuente en lo que respecta a su ubicación, edad de presentación y frecuencia, y es debido a esta razón que consideramos interesante su comunicación debido a que corresponde a un desafío diagnóstico clínico e histopatológico.

\section{CONFLICTO DE INTERESES}

Los autores declaran no presentar conflicto de interés.

\section{BIBLIOGRAFÍA}

1. El-Naggar AK, Chan JKC, Grandis JR, Takata T, Slootweg PJ. (Eds.) WHO Classification of head and neck tumours.4th Edition. IARC: Lyon 2017.

2. Chrcanovic BR, Gomez RS. Calcifying epithelial odontogenic tumor: An updated analysis of 339 cases reported in the literature. J Craniomaxillofac Surg. 2017;45(8):11171123

3. Philipsen HP, Reichart PR. Calcifying epithelial odontogenic tumour: Biological profile based on 181 cases from the literature. Oral Oncol. 2000;(36):17-26.

4. Taneeru S, Guttikonda VR, Korlepara R, Gaddipati R, Kundoor VK. Non calcifying type of calcifying epithelial odontogenic tumor: An unusual case report with special emphasis on histogenesis of calcifications. J Maxillofac Oral Surg. 2017;16 (2):253-257. 5. Ching-Yi Ch, Chung-Wei W, Wen-Chen W, Li-Min L, and Yuk-Kwan Ch. Clear-cell variant of calcifying epithelial odontogenic tumor (Pindborg tumor) in the mandible. Int J Oral Sci. 2013:5 (2):115-119.

6. Hicks MJ, Flaitz CM, Wong ME, McDaniel RK, Cagle PT. Clear cell variant of calcifying epitelial odontogenic tumor: case report and review of the literature. Head Neck. 1994;16 (3):272-7.

7. Afroz N, Jain A, Maheswari V, Ahmad SS. Non-calcifying variant of calcifying epithelial odontogenic tumor with clear cells-first case report of an extraosseous (peripheral) presentation. Eur J Gen Dent. 2013;(2):80-82.
8. Murphy CL, Kestler DP, Foster JS, Wang S, Macy SD, Kennel SJ, et al. Odontogenic ameloblast-associated protein nature of the amyloid found in calcifying epithelial odontogenic tumors and unerupted tooth follicles. Amyloid. 2008;15 (2):89-95.

9. Pindborg JJ. A calcifying epithelial odontogenic tumor. Cancer. 1958;11(4):838-43

10. Mohtasham N, Habibi A, Jafarzadeh H, Amirchaghmaghi M. Extension of Pindborg tumor to the maxillary sinus: a case report. J Oral Pathol Med. 2008;(37):59-61.

11. Priya $S$, Meghanand T, Abhishek $S$, Jayanti $S$. Clear cell calcifying epithelial odontogenic (Pindborg) tumor involving the maxillary sinus: A case report and review of literatura. J Oral Maxillofac Pathol. 2012;16(3):454-459.

12. Misra SR, Lenka S, Sahoo SR, Mishra S. Giant pindborg tumor (calcifying epithelial odontogenic tumor): An unusual case report with radiologic-pathologic correlation. J Clin Imaging Sci. 2013;3(Suppl 1):11.

13. Chaudry AP, Holte NO, Vickers RA. Calcifying epithelial odontogenic tumor: report of a case. Oral Surg Oral Med Oral Pathol 1962;(15):843-7.

14. Rani V, Masthan MK, Aravindha B, Leena S. Aggressive calcifying epithelial odontogenic tumor of the maxillary sinus with extraosseous oral mucosal involvement: $A$ case report. Iran J Med Sci. 2016; (2): 145-149.

15. Patiño B, Fernández-Alba J, Garcia-Rozado A, Martin R, López-Cedrún JL, Sanromán B. Calcifying epithelial odontogenic (Pindborg) tumor: A series of 4 distinctive cases and a review of the literature. J Oral Maxillofac Surg. 2005;(63):1361-1368. 